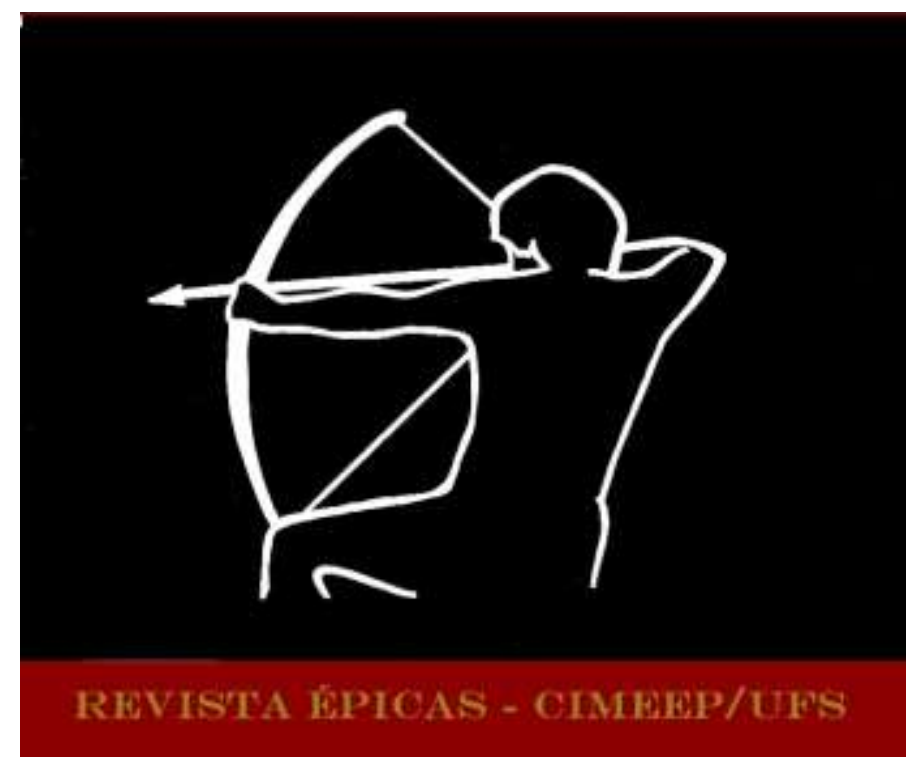

DIALLO, Amadou Oury. Mutations et métamorphoses de l'épique au Fouta-Djalon. In: Revista Épicas. Ano 4, N. 7, Jun 2020, p. 1-13. ISSN 2527-080-X.

\title{
MUTATIONS ET MÉTAMORPHOSES DE L’ÉPIQUE AU FOUTA-DJALON
}

\author{
MUTAÇÕES E METAMORFOSES DO ÉPICO EM FOUTA-DJALON
}

Amadou Oury Diallo

Université Assane Seck, Ziguinchor, Sénégal

\begin{abstract}
RESUME: L'épique connaît au Foûta-Djalon trois âges déterminés par l'évolution historique de la société. Cette évolution est le point nodal des mutations et des métamorphoses du genre. Dans le contexte traditionnel, les poètes épiques restent fidèles aux canons classiques et exaltent les héros ayant marqué la théocratie. Pour leur part, quelques lettrés et érudits célèbrent les figures saintes de l'Islam à travers des épopées hagiographiques et savantes. Dans les nouvelles épopées, le genre survit en se renouvelant et en s'adaptant au contexte : les récits sont empreints de satires et de parodies. La poésie traditionnelle et la musique apparaissent comme les nouveaux espaces où chevauche l'épique. Mots-clés : Fouta-Djalon ; Épopées hagiographiques ; Musique.
\end{abstract}

RESUMO: A epopeia em Fouta-Djalon conhece três idades determinadas pela evolução histórica da sociedade. Essa evolução é o ponto nodal das mutações e metamorfoses do gênero. No contexto tradicional, os poetas épicos permanecem fiéis aos cânones clássicos e exaltam os heróis que marcaram a teocracia. Por sua parte, alguns letrados e eruditos celebram as figuras sagradas do Islã através de epopeias hagiográficas e savantes. Nas novas epopeias, o gênero sobrevive renovando-se e adaptando-se ao contexto: as histórias estão cheias de sátiras e paródias. A poesia e a música tradicionais aparecem como os novos espaços onde se situa o épico.

Palavras-chave: Fouta-Djalon; Epopeias hagiográficas; Música.

\section{Introduction}

L'épopée est, de façon générale, intimement liée à l'histoire et au mythe. Les théoriciens partent souvent de cette double dimension pour établir la classification des récits épiques (MADELENAT, 1986, KESTELOOT, 1997, DERIVE, 2002). Pour le cas du Foûta-Djalon, les récits mettent en avant la dimension historique. L'histoire impériale ${ }^{1}$ fournit à l'épopée sa matière et détermine pour une grande part son

\footnotetext{
${ }^{1}$ Le Foûta théocratique fut fondé en 1725.
} 
évolution. L'épopée comme performance littéraire qui exalte la mémoire collective se définit autant par l'ancrage historico-mythique que par la situation du griot, poète épique.

L'évolution de la société semble être à l'origine de celle du genre épique. Les mutations ainsi que les métamorphoses de l'épique sont d'abord dues aux bouleversements sociopolitiques occasionnés par la colonisation. La production épique du Foûta-Djalon peut même être classée en trois catégories : les épopées traditionnelles, les épopées savantes et les nouvelles épopées.

Dans cette étude, les mutations et les métamorphoses de l'épopée seront envisagées à travers ces trois âges de l'épique. Mais il faut dire que la situation du griot constitue un point nodal qui détermine toute l'évolution du genre. Nous brosserons d'abord le contexte historique du griot, nous mettrons ensuite en lumière les conséquences des bouleversements sociopolitiques, puis nous passerons en revue les mutations et les métamorphoses de l'épique dans les différentes catégories susmentionnées.

Notre réflexion va se baser sur un large corpus épique allant des productions narratives ${ }^{2}$ aux compositions musicales ${ }^{3}$, en passant par l'hymne national'.

\section{Le griot}

Au Foûta-Djalon, comme dans bon nombre de sociétés africaines, le griot est le producteur attitré et spécifique de l'épopée. Lilyan Kesteloot et Bassirou Dieng notent que « la seule condition nécessaire pour qu'il y ait production d'épopée dans des sociétés orales était l'existence d'artistes spécialisés, dont le métier était la mémoire, la conservation, la récitation, l'exaltation des hauts faits de princes ou héros nationaux (...) » (KESTELOOT et DIENG, 1997, p. 52).

L'énonciateur de l'épopée appartient à une catégorie sociale endogamique appelée awluube comportant trois sous-catégories bien hiérarchisées : les ñamakalaabe, les jeliibe et les farbaabe.

Les ñamakalaabe sont des guitaristes et des chansonniers ambulants qui évoluent au gré des cérémonies sociales dont ils sont les animateurs. Les jeliibe, eux, occupent une position intermédiaire. Spécialistes de la musique, ils jouent les instruments tels que le balafon (balan), la guitare à trois cordes (goni), la guitare à quatre ou cinq cordes (keroona), ou la harpe-luth (koora). Les grands musiciens d'aujourd'hui sont souvent issus de ce groupe.

Les farbaabe occupent, pour leur part, le niveau le plus élevé et côtoient l'aristocratie. Alfâ Ibrâhîm Sow les décrit comme " des lettrés qui ont étudié et traduit le Coran, en ont fait l'exégèse, connaissent la théologie et le droit musulmans, participent aux discussions littéraires auprès des autres lettrés de la société, généralement à la mosquée de la province après la prière du vendredi. Attachés aux familles oligarchiques des almâmis et des alfâs, ils suivaient ces chefs à travers leurs déplacements, ne jouaient jamais d'un

\footnotetext{
${ }^{2}$ Amadou Oury Diallo, 2009, Épopée Foûta-Djalon, la chute du Gâbou, Paris, L'Harmattan ; Maladho Siddy Baldé, 2016, L'épopée de Bokar Biro selon Farba Kéba Sow de Labé, Paris, L'Harmattan; Amadou Oury Diallo, 2014, L'enfant prodige, inédit ; Les almâmis de la maison des Soriyâ par Farba Sek (pp. 54-83) et Les Diallo du Labé par Farba Ibrâhîm ", (pp. 84-135) in Alfâ Ibrâhim Sow, 1968, Chroniques et récits du Foûta Djalon, Paris : Librairie C. Klincksieck.

3 Baaba Maal, 1999, L'Épopée d'El-Hadj Omar Taal, Le Ndiambour.

${ }^{4}$ Les hymnes guinéen et malien.
} 
instrument ${ }^{5}$, ont des élèves et des disciples parmi les autres griots ou awluube (gawlo au sing.) et portent le titre de farba ou maître-griot " (SOW, 1968, p. 11). Ils sont les maîtres de la parole publique, politique et poétique. Ils exercent également les fonctions de conseillers, d'historiographes, de généalogistes et d'ambassadeurs.

Comme régulateur social, le griot ou farba (sing. de farbaabe) est investi de fonctions considérables. Ils sont les véritables spécialistes de l'épopée, du mythe, de la généalogie épique, etc. Ils sont les détenteurs de la tradition historico-mythique et de la performance épique. Eu égard à toutes ces fonctions, les farbaabe exercent une grande influence sur les affaires du royaume. Par exemple, évoluant à la cour royale et auprès des dignitaires, qui les entretiennent en échange de services, ils exercent, comme dit Barry (2004), un poids moral considérable sur les mœurs politiques et sociales dans la vie de la cité.

L'origine historique des griots peuls suscite une certaine interrogation : sont-ils peuls ou non ? Pour Christiane Seydou, les griots du Mâcina sont d'origine étrangère : « L'épopée est, chez les Peuls, l'apanage d'une catégorie particulière de griots, les maabuube, qui sont aussi des tisserands. Les Peuls qui, dans leur société nomade traditionnelle, n'ont pas d'artisans " castes ", ont adopté, une fois sédentarisés, les classifications des populations qu'ils côtoyaient; et tout en continuant de considérer les griots comme originellement non-Peuls, ils les reconnaissent comme les détenteurs attitrés de leur patrimoine épique (...) » (SEYDOU, 1988, p. 19). De son côté, Thierno Diallo affirme que les jeliibe du Foûta-Djalon viennent du Manding et les awluube du Boundou et du Fouta Tôro (DIALLO, 1971, p. 90). Ce point de vue est corroboré par les renseignements fournis ${ }^{6}$ par la veuve de Farba Ibrâhîma Ndiâla (Seck). D'après cette source, les grands-parents des awluube, venus du Boundou, se sont d'abord s'installés à Koumbiya dans le Gaoual puis à Timbo, la capitale du Foûta théocratique. Les patronymiques des awluube du Foûta-Djalon sont Niane, Sow, Dieng, Seck, Mbaye, Thiam et Diop.

Quant à l'origine mythique des griots, selon plusieurs versions, c'est au cours d'un voyage de deux frères dans la haute brousse que le cadet, tenaillé par la faim, se plaignit auprès de son grand frère qui, partit lui chasser quelque gibier, n'en trouva pas et, pour ne pas faillir à son devoir d'aîné, coupa une partie de sa cuisse qu'il grilla et offrit à l'affamé pour apaiser sa faim. Ne cessant d'être questionné sur les causes de sa démarche boiteuse, l'aîné finit par avouer. Touché par ce geste, le cadet décida d'être son laudateur en se constituant griot. C'est ainsi que les mythes expliquent la naissance du griot dans la société peule (NGAIDE, 1978, p. 171, SOW TÉLÉMAQUE, 1916, p. 275-278).

La fonction du griot est héréditaire. Un griot pour être reconnu doit effectuer une formation pendant de longues années. Selon Farba Abbâssi Seck, le jeune apprenti commence par suivre son maître dans ses différentes pérégrinations au gré des cérémonies. Au cours de ces déplacements, il apprend à écouter, à mémoriser, à comprendre les protocoles régissant les cérémonies et à se familiariser avec les personnages

\footnotetext{
${ }^{5}$ En fait, c'est là une erreur de la part de Sow. Le Farba peut bien jouer du hoddu (luth), par lequel il accompagne sa narration ; il peut arriver qu'un autre joue et l'accompagne.

${ }^{6}$ Entretien avec Bintou Râbi Seck, Conakry, mars 2018.
} 
importants. Chaque performance de son maître au cours d'un événement est pour lui une occasion d'apprentissage à mettre à profit. L'histoire et la connaissance de la société (la structuration sociale, les origines, la généalogie des familles, les relations entre les lignées, les personnages importants, les secrets et les tabous, etc.) sont les premiers rudiments que le jeune griot assimile. Parallèlement à cette formation, il apprend les sciences religieuses pour pouvoir commercer dans cette société où le pouvoir et son exercice sont définis avant tout par la religion. Il acquiert ainsi l'usage de l'écriture qui vient se greffer à l'oralité.

L'existence et la fonction du farba étaient intimement liées au pouvoir en place. Cet état de fait explique l'évolution du genre dans la plupart des cultures africaines, en particulier au Foûta-Djalon. Du fait du colonialisme, les sociétés africaines ont subi de bouleversements profonds, dont le plus important est la déstructuration sociopolitique qui a démembré la société. Les conséquences ont été dramatiques pour les farbaabe qui, après la disparition des chefferies traditionnelles, se retrouvèrent comme un poisson hors de l'eau. La disparition des royaumes et des empires entraîna celle de nombreux farbaabe. Le peu qui en restait survécut tant bien que mal. La transmission et la continuité du métier qui, rappelons-le, sont héréditaires, se sont estompées dans de nombreux cas. Au lendemain de bataille de Pétel Djiga (le rocher des vautours) le 14 novembre 1896 à Porédaka qui marque la fin de la théocratie du Foûta-Djalon, la situation des poètes épiques change radicalement. C'est ainsi que, par exemple, Farba Moûmini Seck vivant jusque-là à Timbo, la capitale, dût aller se fixer en Sierra Léone ${ }^{7}$, où il a continué son œuvre au milieu de la diaspora peule. Son fils, Farba Ibrâhîma Ndiâla, y a appris les arcanes du métier et y a produit l'Épopée du Foûta-Djalon.

\section{Les épopées traditionnelles}

Il convient d'abord de faire un rappel théorique sur les éléments constitutifs de l'épique. Si le genre peut avoir certaines variations relatives au temps et à l'histoire des sociétés, il existe des traits spécifiques qui déterminent la poétique des récits. Ces traits sont généralement l'emploi du mètre héroïque (pour l'épopée occidentale), le caractère narratif du poème, la composition étendue autour d'une action « entière et complète ", sous forme d'épisodes avec des péripéties merveilleuses et imitant des actions de haute valeur morale (KESTELOOT, 1971, p. 21).

Pour Daniel Madelénat l'épopée se définit et se distingue en tant que parole qui se forge dans la récitation orale, une performance mettant en scène un poète-exécutant, narrateur omniscient et objectif, en face d'un auditoire réactif ; sa composition est marquée par une unité et une complexité épisodique, une syntaxe paratactique et une rhétorique formulaire et hyperbolique (MADELENAT, 1986, p. 23-24).

À côté de ces traits, il faut évoquer le contexte de production et d'énonciation de l'épopée au FoûtaDjalon. L'épopée en tant que récit des faits ou gestes héroïques est essentiellement liée à la guerre. Ainsi les farbaabe entouraient-ils les combattants dans les veillées d'arme en galvanisant leur fibre patriotique par la puissance rhétorique du discours épique. Comme gardien des exploits héröques, ils accompagnaient les

\footnotetext{
${ }^{7}$ Entretien avec Bintou Râbi, la veuve de Farba Ibrâhîm Ndjâla, Conakry, mars 2018.
} 
guerriers dans les champs de bataille pour noter leurs moindres faits et gestes afin de relater plus tard le déroulement de la guerre, mais surtout pour flatter leurs égos et accroître leur courage devant les troupes adverses. L'épopée s'énonçait aussi dans des circonstances paisibles telles que les fêtes d'intronisation des rois ou des chefs de province, les cérémonies funèbres pendant lesquelles les poètes déclament, sous forme d’oraison, la généalogie épique du défunt, etc.

Ce contexte énonciatif est celui de l'épopée classique. Après la colonisation, la donne a changé. Les poètes ont trouvé de nouveaux cadres pour leur performance. Le cas du récit Épopée du Foûta-Djalon est très illustratif du changement advenu. Ce récit s'ouvre ainsi :

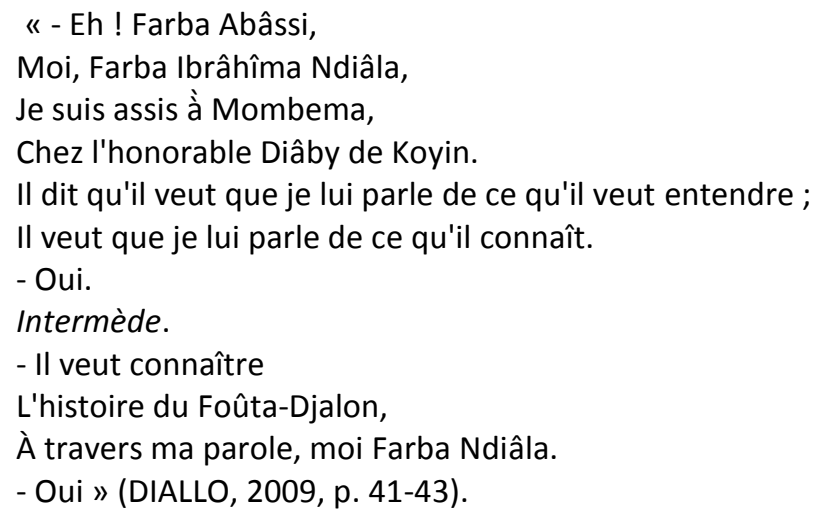

Dans cet incipit, le poète épique, Farba Ibrâhîma Ndiâla, met en place le protocole narratif avec ses différentes instances : énonciateur, répondeur, auditoire, temps et lieu. Par sa dimension spatio-temporelle, ce protocole narratif différencie du contexte traditionnel d'avant la colonisation. En effet, au lieu d'être énoncée dans les circonstances précédemment décrites, l'épopée est dite au cours d'une veillée organisée chez l'honorable Diâby.

D’après Farba Abbâssi, le griot qui fait office de répondeur au récit, l’honorable Diâby de Koyin, un millionnaire enrichi dans l'extraction de pierres précieuses (or et diamant), avait invité Farba Ibrâhîma Ndiâla à son domicile à Mombéma, en Sierra Léone. La veillée devait avoir lieu dans son salon, mais il y eût tellement de monde qu'elle fut déplacée dans la cour. Le récit fut enregistré, pour la première fois, durant cette veillée. La " prestation » du griot eut un succès immense et sa performance se diffusa grâce aux cassettes comme une trainée de poudre au sein de la diaspora peule du Foûta-Djalon de Sierra Léone. Quelques temps après, son œuvre franchit les frontières du pays et devient populaire dans les autres milieux peuls.

Ordinairement, la veillée est le moment propice de l'énonciation du conte, mais avec le changement intervenu dans la société, elle l'est aussi pour l'épopée. Le fait que l'épopée soit enregistrée est en soi une nouveauté. Si la fixation fossilise l'œuvre, elle la sauve néanmoins de la perdition, modifie les conditions de sa réception et de son actualisation. Grâce à sa variabilité, l'œuvre orale est appelée à une perpétuelle récréation, chaque actualisation se différenciant des précédentes, les modifications allant jusqu'à estomper l'identité de l'œuvre (Zumthor, 1983, p. 245). Mais à partir du moment où l'œuvre sort de la bouche de son interprète ou exécutant pour être domestiquée dans un support (disque, cassette, magnétophone, etc.), il y 
a une séparation non seulement entre l'artiste et son œuvre, mais également entre l'artiste et son auditoire. Désormais, l'artiste n'a plus le moyen d'interagir avec l'auditoire ni celui d'intervenir hic et nunc sur son œuvre comme il le faisait durant une performance authentiquement orale. Analysant le rôle des médias dans l'évolution de la performance, Paul Zumthor écrit : « disque, magnétophone, cassette ou radio, les médias auditifs tendent à éliminer, avec la vision, la dimension collective de la réception. En revanche, ils touchent individuellement un nombre illimité d'auditeurs. (...) La performance s'est intériorisée. En dépit de l'usage que l'on en fait couramment en groupe (spécialement en vue de la danse), ces médias s'accordent mieux ainsi à l'audition solitaire et, le cas échéant, critique » (ZUMTHOR, 1983, p. 238).

L'enregistrement de l'épopée modifie complètement les modalités de réception du récit. De performance théâtralisée, vivante et donnant la possibilité d'une interaction entre exécutant et public, l'œuvre, finie et immuable, s'offre une fois pour toutes.

Il faut dire enfin que ces épopées traditionnelles demeurent vivantes dans la mémoire collective et font souvent l'objet d'actualisations et d'adaptations par la poésie orale et la musique qui se nourrissent de la sève épique.

La poésie orale - les chants populaires et les hymnes principalement - constitue un lieu de refuge important de l'épique en Afrique noire. Ce n'est pas un hasard si les hymnes nationaux du Mali, de la Guinée et d'autres pays proviennent d'airs épiques, donc de la poésie épique : Soundiata Kéita ${ }^{8}$ et Alpha Yâya Diallo. L'écrivain Seydou Badian Kouyaté qui a composé l'hymne national malien affirme s'être « inspiré d’un air du XIII siècle, un air qui figurait dans l'épopée de Soundiata Keita (fondateur de l'empire du Mali) " (PERRET, 2010). Quant à l'hymne guinéen, il est né dans des circonstances particulières. Convoqués à Conakry pour une conférence nationale par le Gouverneur Ballay qui voulait s'assurer leur collaboration, tous les chefs locaux de la Guinée vinrent de partout. Alpha Yaya, lui, arriva le dernier « avec une escorte incroyablement étoffée autant qu'imposante et dans un faste inégalé. Sa suite répartie en six groupes fit son entrée à raison d'un groupe par jour, afin de tenir la capitale en émoi admiratif. Le sixième jour, à la tête d'un cortège composé de notables, de marabouts et de griots (tous à cheval) chantant ses louanges, il fit lui-même son entrée, caracolant sur un superbe coursier blanc, unique en son genre » (SANO, 1963). Alpha Yaya ravit ainsi la vedette à tout le monde :

Conakry n'avait d'yeux et d'oreilles que pour le « prince charmant » dont le faste, la générosité et la singulière fascination étaient devenues le thème des conversations courantes ". II s'en est fallu de peu que la conférence passât au second plan tellement le roi peul avait cristallisé l'attention autour de lui. C'est dans ce climat euphorique que le griot Korofo Moussa, venant de Kissidougou, « décida d'aller saluer et amuser « I'homme du jour » qu'il trouva majestueusement assis sur une chaise dominant la foule bigarrée accourue à ses côtés » (SANO, 1963).

C'est dans ces circonstances que Korofo Moussa improvisa un chant en l'honneur d'Alpha Yaya. Le chant connut une fortune incroyable puis que « à peine né, l'air d'Alpha Yaya fut appris, chanté et joué par tous les griots descendus à Conakry pour la conférence. En quelques jours, la capitale toute entière - foyer

8L'épopée de Soundiata a donné l’hymne national du Mali, et celle du Kajoor, le chant de la Jeunesse du Sénégal, p. 66, cf. L. Kesteloot, Les épopées d'Afrique noire, op. cit. et Christiane Seydou, "épopée et identité », art. cit., p. 14. 
de rayonnement - le fredonna. Dibi, le griot personnel d'Alpha Yaya s'entraîna aussitôt à l'exécuter dans le meilleur style (...) Et au retour des délégations dans leurs régions respectives, comme une traînée de poudre le chant se répandit rapidement de proche en proche jusque dans nos moindres villages » (SANO, 1963). C'est ainsi qu'est né l'hymne national guinéen.

\section{Les épopées savantes}

Après les épopées traditionnelles, la deuxième phase d'évolution de l'épique correspond à des œuvres qui se définissent par leur caractère savant et qui sont élaborées généralement par des érudits : prédicateurs illuminés, lettrés musulmans ou maîtres griots. Les épopées de cette catégorie concernent les figures prophétiques ou saintes (Mahomet, Idris, David, Ali, Housseyni, etc.) et appartiennent à la veine religieuse ou mystique. Elles forment un cycle centré sur le thème de la sainteté. Énoncés tantôt en arabe suivi de sa traduction en pulaar, tantôt en pulaar, ces récits s'inspirent de l'hagiologie islamique et allient l'écriture à l'oralité. Ils sont produits par des conteurs érudits qui adoptent souvent une posture d'exégète, de moraliste et d'historiographe. Le passage suivant décrit le combat entre Ali, le cousin du Prophète, et Amrou, dans la célèbre bataille de Khaybar opposant Mahomet et Abû Jahl, le " père de l'ignorance » :

Ils se soulevèrent, ils se.... Une... vilaine. Amrou vit que son élan ressemblait à celui d'un grand épervier qui plonge pour ravir des poules. II dit à sa copine de descendre et de s'éloigner car celui qui fonçait sur lui n'allait pas le manquer. (...) Amrou s'élança, Badara ${ }^{9} s^{\prime}$ élança, ils se cognèrent rap ! D’où ils venaient, les gens émirent de grands cris. Une nuée de poussière s'éleva autour d'eux tel un brouillard. Les mécréants prièrent leurs idoles pour que la poussière disparût et qu'ils eussent des nouvelles d'Amrou, les croyants prièrent Allah et son Prophète pour que la poussière disparût et qu'ils eussent des nouvelles du seigneur Ali. La poussière disparut. Ils virent que personne n'avait même pas enlevé un seul cheveu à l'autre. Leur empoignade ressemblait à un choc d'éléphant dans la forêt. Après, Badara s'élança de nouveau, Amrou s'élança, ils se cognèrent rap! D'où ils venaient, les gens émirent de grands cris. Amrou souleva très haut une lance qu'il brandit et qu'il projeta contre le seigneur Ali. Celui-ci tira son destrier, le fit cabrer, la lance se flanqua sur terre. Badara se posa sur terre et s'apprêta à lui rendre le coup. Lui aussi brandit la lance, la souleva haut et la projeta contre Amrou. Celui-ci se mit à imiter son action : son cheval à lui se cabra, mais la lance d'Ali se planta dans sa poitrine, la fendant en deux parties qui s'abattirent chacune d'un côté. Amrou se releva et lui dit : "Attends que je cherche un autre cheval puisque tu as tué le mien ». Le seigneur Ali répondit : " Je ne te poursuivrai que quand tu mettras trop de temps à revenir ; tu ne me trouveras pas ailleurs qu'ici. (...)

Dans sa fuite, Amrou rencontre Satan qui, se faisant passer pour un messager divin envoyé à sa rescousse, le convainc de continuer le combat :

Amrou croisa Satan qui portait un grand boubou, coiffé du bonnet de l'hypocrisie et tenant une canne. Il lui demanda : "Où vas-tu ? " - C'est de celui-là dont m'avait prévenu ma mère dans sa divination ». Satan lui dit : « C'est moi qu'on appelle l'ange Gabriel. C'est Dieu qui m'envoie pour te dire de persévérer car tu vas bientôt vaincre Ali. (...) ».

II s'élança et vint. Le seigneur Ali l'aperçut-il venir, qu'il quitta son destrier et le trouva sur le sien. Il s'agrippa à sa main droite et la tordit si bien qu'il ouvrît grande sa bouche et montrât ses dents. Amrou lui dit : "Si tu ne lâches pas prise, mon âme en sortira de sitôt ". Il lui répondit : " Je ne relâcherai point; dis ce que tu n'as jamais dit pour que je te laisse en vie ${ }^{10}$. Amrou dit : "Point de divinité si ce n'est Allah ». Ali tint fermement sa gorge et lui : « Tu n'achèveras pas cette parole

\footnotetext{
${ }^{9}$ Surnom affectif d'Ali.

${ }^{10} C^{\prime}$ est-à-dire : « professe la foi ».
} 
car en te tuant alors, j'aurais tué un croyant ". Il prit le pommeau de son sabre et s'apprêtait à tuer Amrou, mais Allah ne le permit pas ${ }^{11}$. Amrou dit : "II ne convient point de remercier Allah, mais plutôt demander d'être absout ". Ali tira le sabre, mais vit qu'il ne bougeait pas. Il se saisit d'Amrou par ses dents, le traina une bonne distance. Il enlevait de son dos des lambeaux de chair d'une longueur d'un empan qu'il jetait par terre, Amrou finit par dire : " Je te demande pardon ! Je te demande pardon! » Ali lui rétorqua: "Me demandes-tu pardon avant Dieu et son ami ? " (DIALLO, 2020).

Les récits de cette veine islamique sont généralement courts et portent sur des épisodes choisis, rarement sur le parcours entier des personnages concernés. Le Coran et l'hagiologie prophétique constituent le substrat narratif de ces récits que le caractère érudit et la dimension rendent ésotériques, voire mystiques et moins accessibles au public n'ayant pas une certaine culture arabo-islamique.

\section{Les nouvelles épopées}

La situation qui prévaut à la fin de la théocratie conduit l'épopée à se renouveler et à s'adapter au contexte nouveau. Sous la colonisation, l'épopée entretient le souffle patriotique et devient le refuge des peuples vaincus. L'exaltation des héros du passé devient quelque chose d'apaisant qui apporte une certaine consolation. C'est ainsi que l'on peut

considérer l'épopée comme une des modalités possibles de la mise en œuvre du processus par lequel les communautés humaines transforment leurs défaites en des sortes de victoires. Non pas des victoires sur les autres (ils acceptent les défaites et le disent), mais plutôt sur eux-mêmes, en se sublimant à travers leurs héros (LY, 2002, p. 245).

Les épopées traditionnelles sont réactualisées, les gloires du passé convoquées comme des motifs de consolation ou de refuge par rapport aux jougs du présent : le souvenir des héros du passé et de leurs exploits constitue une source d'inspiration et de réconfort pour les peuples dominés. Les figures épiques traditionnelles ont joué un rôle fédérateur et unificateur dans les colonies. Ce n'est pas un hasard si, après les indépendances, les travaux universitaires et la publication d'épopées a connu un essor considérable en Afrique.

Mais les récits qui relèvent à proprement parler du domaine de la néo-épopée, pour le cas du FoûtaDjalon, sont des œuvres hybrides tenant de l'épopée et d'autres genres tels que le conte, la poésie religieuse et la chronique. Le récit « L'enfant prodige » est à cet égard un cas illustratif.

Cette œuvre, comme les épopées traditionnelles, tire son ancrage de I'histoire théocratique, notamment des luttes intestines qui opposèrent les deux branches dynastiques (soriya et alphaya) entre 1781 et 1793. Cet épisode des tensions internes, point de départ de la fiction narrative et toile de fond, n'est qu'un prétexte parce que l'objet réel du récit est les événements des années 1980-90. Farba Ibrâhîma Ndiâla, chef de file des maîtres griots et grand producteur d'épopées, a vécu les événements tragiques de la guerre civile en Sierra Léone. II dénonce à travers L'enfant prodige les troubles qui naissent de la confiscation du

\footnotetext{
${ }^{11}$ Le sabre refusa de sortir.
} 
pouvoir. C'est là un changement radical et une évolution inédite de l'épique parce que, jusque-là, le ton de l'épopée est plutôt encomiastique, mais en mettant en scène un matamore qui brille par ses excès, le poète renverse la logique narrative de l'épopée traditionnelle en parodiant le genre et en y greffant subtilement les traits du conte animalier comme la façon de nommer les personnages (Mamadu mo hulataa : MamadouI'intrépide, Boobo Malaado : L'enfant bienheureux, Faatumata-biidaane : Fâtoumata-la-génie). Le fait aussi que l'enfant prodige, soit élevé et initié par un djinn dans une grotte est un motif de la fable animalière. Ajoutons à cela l'indétermination spatio-temporelle de l'intrigue narrative qui est ordinairement une des propriétés du conte. L'incipit du récit est, à ce propos, très évocateur :

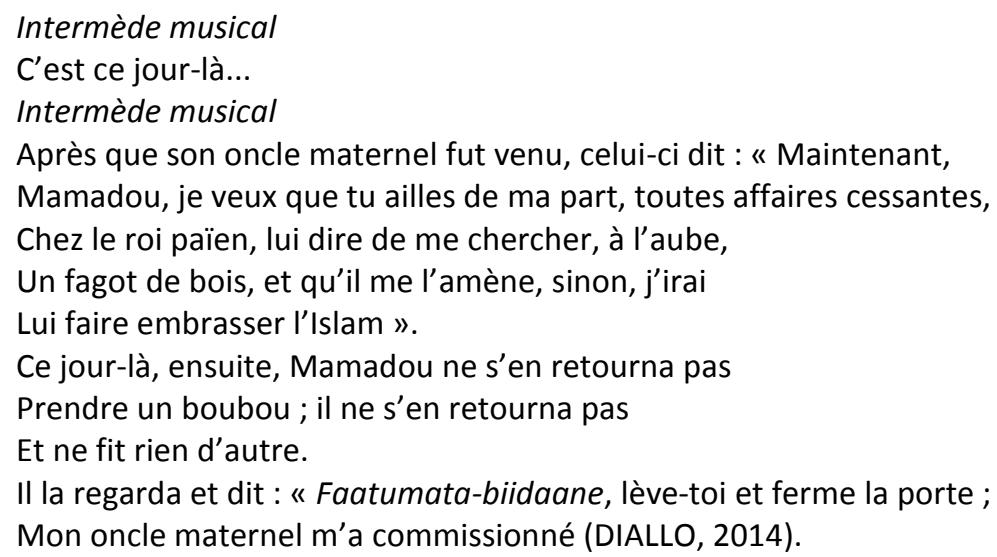

Pour s'apparenter au conte, le récit de L'enfant prodige n'en garde pas moins les traits épiques dont les plus saillants sont l'air musical et les prouesses dont Boobo Malaado fait montre tout au long de l'histoire. Il y a ainsi dans ce récit une sorte d'hybridation de l'épopée et du conte ; cette hybridation s'opère au moyen d'une transformation qui efface certains traits épiques tels que le protocole énonciatif et le cadre spatiotemporel dont nous avons déjà évoqué les effets narratifs.

Par ailleurs, le fait que le griot adopte une posture de satiriste est une des facettes de cette néoépopée. Si l'épopée traditionnelle exalte les actes de bravoure d'un héros désintéressé qui se bat moins pour sa gloire personnelle que pour celle de l'honneur de la patrie, avec Boobo Malaado les choses sont tout autres : la gloire personnelle vient avant toute autre chose. L'appât du gain s'avère être ce qui le motive dans sa lutte de reconquête du pouvoir. Après sa victoire contre son oncle, Boobo Malaado se fait couronner pendant une cérémonie fastueuse inouïe : il fait orner son itinéraire de chaînes d’or et son passage de couvertures de laine (DIALLO, 2014). Sa mégalomanie est telle que, pour se convaincre des délices du pouvoir, il décide de faire porter à dos d'homme les vaches de sa mère depuis leur ancien domicile jusqu'au palais :

Boobo Malaado dit : " S'il est vrai que le pouvoir est agréable, Je veux qu'on porte ma mère et qu'on l'amène ici, Que les vaches qui sont là-bas soient portées et amenées ici. Tout ce que j'ai laissé chez nous, je veux Que rien ne vienne ici de soi-même (DIALLO, 2014). 
Tel est Boobo Malaado, personnage singulier qui, par sa démesure, apparaît comme un héros épique à qui il manque toutefois certains attributs pour ressembler trait pour trait au héros de l'épopée traditionnelle. Comme attributs lui faisant défaut, il y a la noblesse morale et le désintéressement. La noblesse morale est un caractère épique, une grandeur d'âme dont le héros fait preuve en toute situation. Elle interdit les actes qui avilissent ou rabaissent moralement devant l'opinion générale. Dans l'épisode de sa rencontre avec le vieil homme qui avait l'habitude de ne pas répondre aux salutations, Boobo Malaado l'injurie et l'humilie devant ses fils :

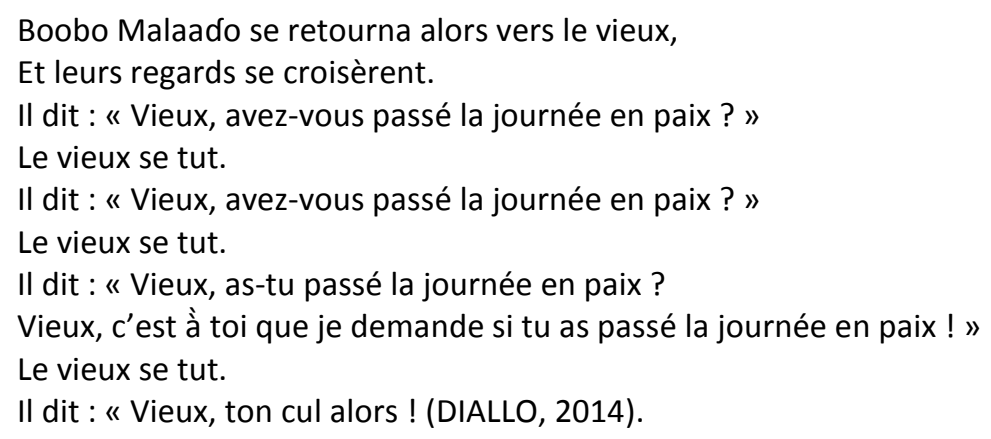

Par ces actes d'irrespect, de grossièreté et d'abus de la force, Boobo Malaado apparaît comme un avatar dégénéré du héros épique traditionnel. L'évolution de l'épique a entraîné une métamorphose de la figure du héros. Comme sa visée est satirique, le récit de L'enfant prodige procède à une parodie de l'épique pour mieux dépeindre les nouvelles figures du pouvoir de l'Afrique contemporaine. Ces nouvelles figures de héros sont présentées comme des matamores et des contres modèles.

En outre, aujourd'hui, si l'épopée survit, c'est grâce surtout à la musique et à la poésie orale qui reprennent à leur compte quelques figures héroïques et épisodes épiques. Ces deux genres participent à entretenir la mémoire et le souffle épiques. C'est comme si on assistait à une migration de l'épique dans la musique traditionnelle et moderne. De fait, du début des indépendances à nos jours, les récits épiques consacrés à des personnages historiques tels que Soundiata, Samba Guéladiégui, Samory Touré ${ }^{12}$, Alpha Yaya, Almâmy Oumar, Bocar Biro, Elhadj Omar ${ }^{13}$, Cheikh Ahmadou Bamba et Lat Dior sont repris par les musiciens dans des chansons qui rappellent et exaltent leurs exploits hérö̈ques. Baaba Maal, un des grands chanteurs peuls, ouvre comme suit son album musical «L'Épopée d'El-Hadj Omar Taal » dont le titre est évocateur :

\footnotetext{
« Mesdames et Messieurs, à l'occasion du bicentenaire de la naissance d'El Hadj Omar Taal, le " Daade Leñol » a composé ce titre en guise d'hommage respectueux à la mémoire d'un illustre fils d'Afrique dont la sublime épopée marquera à jamais le souvenir de ses semblables. De sa glorieuse existence rapportée par l'Histoire impartiale, la postérité en retient les traits d'une figure légendaire, l'exemple achevé de l'élévation spirituelle dévouée à la rédemption des peuples et des nations. De nombreuses anecdotes conservées par la tradition magnifient à travers les âges sa singulière destinée qui se manifesta dès l'aube de sa vie » (MAAL : 1999).
}

\footnotetext{
12 Kouyaté Sory Kandia, 1970, L'épopée du mandingue, vol. 1.

${ }^{13} \mathrm{Cf}$. http://chantshistoiremande.free.fr/Html/taara.php
} 
La musique devient ainsi le terrain propice où la mémoire épique trouve son refuge. C'est une évolution naturelle car le chant musical, de par sa nature poétique même, appartient au genre de la poésie orale qui est constituée en grande partie par l'épique et l'héroïque. L'air musical aussi qui est une part essentielle de l'épique est le point de jonction entre la musique et l'épopée. Le fait enfin que les chanteurs soient souvent issus de la catégorie des griots favorise l'échange entre la musique et l'épopée. La musique joue un grand rôle dans la transmission et la conservation de la tradition épique dans la mesure où c'est elle qui permet à la grande masse de la population d'avoir accès à la mémoire des faits héroïques. En réactualisant les faits épiques sous forme de chanson, la musique revitalise le genre dont elle constitue un vecteur essentiel de pérennisation dans la mémoire collective. Elle contribue, par ailleurs, à rendre toujours vivant le souffle épique à travers les airs musicaux qui, loin d'être de simples accompagnements mélodiques, sont très utiles et même indispensables dans la culture peule pour l'exaltation des figures héroöques car rien d'autre ne définit mieux un personnage épique que sa devise musicale (SEYDOU, 1977). Par exemple, l'air musical « tarra » a été repris puis renouvelé par plusieurs chanteurs à travers le temps :

La tradition veut que ce soient les griots Diabaté qui aient renouvelé le chant « Taara »; au tout début, au plus loin que remonte l'Histoire, la « Tarra » fut dédiée à Lamido Dioulbé, chef de Nioro ; mais après la conquête de cette cité, les griots Diabaté renouvelèrent le chant "Taara » en I'honneur d'El Hadj Omar, puis après sa mort, pour Ahmadou, et enfin pour Makki Tall, ses fils les plus célèbres qui furent de farouches résistants à la colonisation française ${ }^{14}$.

Il faut noter, par ailleurs, qu'à l'échelle continentale, de nombreuses autres figures héroïques ont été célébrées par la musique ${ }^{15}$ et même mises en scène par le roman ${ }^{16}$ ainsi que le théâtre ${ }^{17}$ qui contribuent ainsi à les immortaliser.

\section{Conclusion}

L'évolution de l'épique au Foûta-Djalon correspond à trois âges : les épopées traditionnelles, les épopées savantes et les nouvelles épopées. Le changement socio-politique est le facteur déterminant des mutations et des métamorphoses de l'épique. Si les bouleversements de la structure de la société traditionnelle ont fortement impacté sur la situation du griot, celui-ci a néanmoins pu survivre durant une certaine période, adapter son œuvre et adopter de nouvelles attitudes par rapport à la nouvelle donne.

\footnotetext{
14 http://chantshistoiremande.free.fr/Html/taara.php

${ }^{15}$ Amy Koita, Best Of - vol. 1 - Sazima/Sonima - ref. : SMCD1408 (le titre « Hampaté Bah » est une composition fondée sur le thème de "Taara »; Sidiki Diabaté, D. Sissoko, B. Kouyaté : Cordes Anciennes, 1970 ("Sundjata », " Tara »; Kouyaté, Sory Kandia, 1970 et 1973, L'épopée du Mandingue, vol. 1 et 2, Label : Éditions Bolibana.

16 Diop, Boubacar Boris, 2010, Le cavalier et son sombre, Paris, Philippe Rey; Diop, Boubacar Boris, 1980, Les tambours de la mémoire, Paris, L'Harmattan ; Seck Samb, Rahmatou, 2016, Fergo : tu traceras ta route, Dakar, Abis.

${ }^{17}$ Abdou Anta Ka, 1972, Les Amazoulous, Paris, D.A.E.C ; Condetto Nénékhaly-Camara, 1970, Continent-Afrique suivi de Amazoulous, P.J. Oswald ; Djibril Tamsir Niane, 2009, Sikasso ou la dernière citadelle, Abidjan : NEI-CEDA, Conakry : SAEC; Amadou Cissé Dia, 1987, Les derniers jours de Lat Dior, Paris, Présence Africaine, Nathan ; Mamadou Seyni Mbengue, 1970, Le procès de Lat Dior, Paris : D.A.E.C. ; Bernard Dadié, 1970, Béatrice du Congo, Paris, Présence africaine et Cheikh Aliou Ndao, 1973, Le fils de l'almami, Paris, L'Harmattan - Oswald et Senghor, Léopold Sédar, 1990, « Chaka » CEuvre poétique, Paris, Seuil, pp. 122-137.
} 
Les épopées traditionnelles se rapportent à des héros historiques ayant marqué l'histoire impériale. Dans ces récits, la dimension historique prévaut sur celle mythique. Ces œuvres subsistent grâce à la fixation dans des supports magnétiques ou électroniques.

Dans les épopées savantes, les récits du cycle islamique revisitent l'histoire et l'hagiologie de certaines figures saintes et prophétiques. Dites en arabe et en pulaar, ces épopées hagiographiques sont des productions savantes qui s'adressent davantage à l'élite intellectuelle.

Quant à la néo-épopée, elle est marquée par un ensemble de récits qui s'affranchissent des contraintes ou des règles classiques de l'épopée ; formés à partir d'une hybridation de l'épopée classique et le conte, certaines nouvelles épopées sont très satiriques et témoignent d'un certain engagement de leur « auteur».

Aujourd'hui, les nouvelles formes de l'épique sont à chercher dans la poésie orale et la musique.

\section{Références bibliographiques}

BALDE, Maladho Siddy. L'épopée de Bokar Biro selon Farba Kéba Sow de Labé, Paris : L'Harmattan, 2016. BARRY, Alpha Ousmane. Mode d'expression poétique et stratification sociale dans l'état théocratique du Fouta Djallon. In : Semen, 18, 2004. [En ligne : http://journals.openedition.org/semen/2294].

DERIVE, Jean. L'épopée : unité et diversité d'un genre. Paris : Karthala, 2002.

DIA, Amadou Cissé. Les derniers jours de Lat Dior. Paris : Présence Africaine, Nathan, 1987

DIABATE, Sidiki, D. SISSOKO, B. KOUYATE. Cordes Anciennes. Buda, 1970.

DADIE, Bernard. Béatrice du Congo. Paris : Présence africaine, 1970.

DIALLO, Amadou Oury. Traditions épiques du Foûta-Djalon. inédit, 2020.

DIALLO, Amadou Oury. Histoire et fiction, contextes, enjeux et perspectives : récits épiques du FoûtaDjalon. Thèse unique de $3^{\mathrm{e}}$ cycle, Université Nice Sophia Antipolis, tome 2, inédit, 2014.

DIALLO, Amadou Oury. Épopée du Foûta-Djalon : la chute du Gâbou. Version peule de Farba Ibrâhîma Ndiâla. Paris : L'Harmattan/IFAN-OIF, 2009.

DIALLO, Thierno. Les institutions politiques du Foūta Dyalon au XIX ${ }^{\mathrm{e}}$ siècle (Fii laamu alsilaamaaku Fuuta Jaloo). Dakar : IFAN, 1972.

DIOP, Boubacar Boris. Le cavalier et son sombre. Paris : Philippe Rey, 2010.

DIOP, Boubacar Boris. Les tambours de la mémoire. Paris : L'Harmattan, 1980.

FARBA, Ibrâhîm. Les Diallo du Labé. In : SOW, Alfâ Ibrâhim. Chroniques et récits du Foûta Djalon. Paris : Librairie C. Klincksieck, 1968, p. 84-135.

KA, Abdou Anta. Les Amazoulous. Paris : D.A.E.C., 1972.

KESTELOOT, Lilyan. Les Épopées d’Afrique noire. UNESCO, Karthala, 1997.

KOITA, Amy. Best Of - vol. 1 - Sazima/Sonima - ref. : SMCD1408.

KOUYATE, Sory Kandia . L'épopée du Mandingue. vol. 1 et 2, Label : Éditions Bolibana, 1970/1973.

LY, Amadou, 2002. La victoire des vaincus: l'épopée d'Afrique comme discours compensatoire d'une communauté vaincue. In : Littérales, $n^{\circ} 29$, Paris X-Nanterre, p. 243-252.

MAAL, Baaba. L’Épopée d’El-Hadj Omar Taal. Le Ndiambour, 1999 (Album musical). 
MBENGUE, Mamadou Seyni. Le procès de Lat Dior. Paris : D.A.E.C., 1970.

NDAO, Cheikh Aliou. Le fils de l'almami. Paris : L'Harmattan - Oswald, 1973.

NENEKHALY-CAMARA, Condetto. Continent-Afrique suivi de Amazoulous. P.J. Oswald, 1970.

NGAÏDE, Mamadou Lamine. Étude historique, thématique et stylistique de deux récits peuls. Mémoire de Maîtrise. Université de Dakar, 1978.

NIANE, Djibril Tamsir. Sikasso ou la dernière citadelle. Abidjan : NEI-CEDA, Conakry : SAEC, 2009.

PERRET, Thierry. Objet des indépendances: un hymne national. En ligne : http://www.rfi.fr/fr/contenu/20100305-objets-independances-hymne-national, 2010.

SANO, Mamba. De la mélodie populaire "Alpha Yaya » à l'Hymne national « Liberté ",. In : Recherches africaines, $\mathrm{n}^{\circ}$ 2-3, Avril-Septembre, 1963, p. 28-32, disponible en ligne sous l'Url: http://www.webguinee.net/bibliotheque/archives/rechAfric/1963/2-3/AYHymneNational.html.

SECK SAMB, Rahmatou. Fergo : tu traceras ta route. Dakar : Abis, 2016.

SEK, Farba. Les almâmis de la maison des Soriyâ. In : SOW, Alfâ Ibrâhim. Chroniques et récits du Foûta Djalon,. Paris : Librairie C. Klincksieck, 1968, p. 54-83.

SENGHOR, Léopold Sédar. Chaka. In : Euvre poétique. Paris : Seuil, 1990, p. 122-137.

SEYDOU, Christian. Épopée et identité : exemples africains. In : Journal des africanistes, tome 58, fascicule 1, 1988, p. 7-22.

SEYDOU, Christiane. La devise dans la culture peule : évocation et invocation de la personne. In: CALAMEGRIAULE, Geneviève (dir.). Langage et cultures africaines : essais d'ethnolinguistique. Paris : Maspero, 1977. SOW, Alfâ Ibrâhîm. Chroniques et récits du Foûta-Djalon. Paris : Klincksieck, 1968.

SOW TELEMAQUE, Hamet. Origine des griots. In: Bulletin de I'Enseignement de I'Afrique Occidentale Française, Gorée. Imprimerie du Gouvernement Général, 1916, $4^{\text {è }}$ année, n 25, juin, p. 275-278.

ZUMTHOR, Paul. Introduction à la poésie orale. Paris : Seuil, 1983. 